Article

\title{
Pt-Based Nanostructures for Observing Genuine SERS Spectra of $p$-Aminothiophenol (PATP) Molecules
}

\author{
Yinong Wang ${ }^{1,+}$, Haofei Zhao ${ }^{2,+}{ }^{,}$En Cao ${ }^{2}$, Yinghui Sun ${ }^{2}$, Huanyu $\mathrm{Ye}^{2}$, Weihua Lin ${ }^{2}$ and \\ Rongming Wang ${ }^{2, *}$ \\ 1 Department of Physics, Beihang University, Beijing 100191, China; wangyn.pj@163.com \\ 2 Beijing Key Laboratory for Magneto-Photoelectrical Composite and Interface Science, \\ School of Mathematics and Physics, University of Science and Technology Beijing, Beijing 100083, China; \\ zhaohaofei@ustb.edu.cn (H.Z.); 13573119116@163.com (E.C.); yhsun@ustb.edu.cn (Y.S.); \\ s20160828@xs.ustb.edu.cn (H.Y.); 15501115088@163.com (W.L.) \\ * Correspondence: rmwang@ustb.edu.cn; Tel.: +86-133-9157-1538 \\ + These authors contributed equally to this work.
}

Received: 15 August 2017; Accepted: 9 September 2017; Published: 15 September 2017

\begin{abstract}
By one-pot wet chemical method, Pt-based hollow nanostructures were synthesized at room temperature. Because of the highly damping optical response of the metal, these Pt-based hollow nanostructures exhibited weak thermal effects with a laser focal spot on 4-aminothiophenol (PATP) molecules limiting dimerisation. The isolated surface enhanced Raman scattering (SERS) spectra of PATP, in which the vibrational bands from 4,4'-dimercaptoazobenzene (DMAB) molecules are not observed, were able to be seen, and this was in good agreement with the Raman spectra of PATP powder. In addition, the concentration of PATP molecules was varied, and the illumination time was increased to $2000 \mathrm{~s}$, respectively. It was found that spectra were stable with varied PATP concentrations, and the plasmon-driven chemical conversion of PATP to DMAB was still suppressed, even when the laser illumination time was increased to $2000 \mathrm{~s}$.
\end{abstract}

Keywords: SERS; Pt; hollow structure; PATP

\section{Introduction}

Nanomaterials can exhibit unique properties due to the size effects, when compared to their bulk conterparts [1-5]. Among various nanostructures, the hollow nanostructure has attracted significant attention owing to its high surface area [6], low density, and potential applications in a series of technologies, such as catalysis [7], optical detection [8-11], and photothermal therapy [12]. Therefore, it is of interest to explore hollow-structured nanomaterials in the hope of disclosing their important physical properties.

One of the most significant physical properties of nanostructures is their optical/plasmonic properties [13,14], which is characterized by their strong interaction with resonant photons through the excitation of surface plasmon resonance (SPR). The SPR effect can be utilized in the analytical technique of surface enhanced Raman scattering (SERS) [15-18]. Current accepted knowledge suggests that noble metals $(\mathrm{Au}, \mathrm{Ag}$, and $\mathrm{Cu})$ are the best materials as SERS substrates, with high enhancement factors [19], and therefore they have been widely applied in Raman detection since the discovery of SERS in 1970s [20]. During the past decades, many efforts have been made to break the limitation of substrate specificity and to expand SERS applications to other metals, especially the transition metals $\mathrm{Pd}$ and $\mathrm{Pt}$, which are widely used in catalysis $[15,21,22]$. Recently, it was reported that bimetallic/multiple metallic nanomaterials display novel properties distinct from the pure Pd or Pt element nanostructures [23-28]. 
Unique physical properties benefiting from the synergistic effect of magnetic material ( $\mathrm{Ni}, \mathrm{Co}, \mathrm{Fe})$ alloying were observed in Pt-based bimetallic nanomaterials, such as good electrical, catalytic, and stability characteristics $[29,30]$.

SERS spectra of 4-aminothiophenol (PATP) molecules adsorbed on a SERS substrate surface have been widely investigated. In previous work, bands seen at 1140, 1390, and $1432 \mathrm{~cm}^{-1}$ have been ascribed to the $b 2$ mode, which is selectively enhanced by the chemical enhancement effect [31-33]. It has been confirmed that these peaks come from 4,4'-dimercaptoazobenzene (DMAB) molecules due to the dimerisation reaction from PATP to DMAB by surface catalysis, making the observation of SERS spectrum of PATP molecules a challenge [31]. In this work, we attempt to observe the genuine SERS spectrum of PATP with a novel substrate. By one-pot wet chemical method using water solution at room temperature, Pt-based hollow nanostructures were synthesised. Because of the highly damping optical response of the metal, these Pt-based hollow nanostructures exhibited weak dimerising effects with PATP molecules [34]. The genuine SERS spectra of PATP was able to be seen, where the vibrational bands at 1140, 1390 and $1432 \mathrm{~cm}^{-1}$ from DMAB molecules were absent from observation. Furthermore, the SERS spectra remained consistent with the Raman spectra of PATP powder under laser illumination for more than $2000 \mathrm{~s}$, during the experimental measurement process.

\section{Materials and Methods}

All raw materials in the experiment, such as $\mathrm{K}_{2} \mathrm{PtCl}_{6}(99.8 \%)$, $\mathrm{PVP}(99.5 \%), \mathrm{CoCl}_{2} \cdot 6 \mathrm{H}_{2} \mathrm{O}(25 \%)$, and sodium borohydride (98\%) which were purchased from LANYI REAGENT Ltd. (Beijing, China), were used as-supplied, without further purification. Pt-based hollow NCs were obtained by one-pot wet chemical method. Typically, $\mathrm{CoCl}_{2} \cdot 6 \mathrm{H}_{2} \mathrm{O}(8.5 \mathrm{mg}$ ) and PVP (polyvinylpyrrolidone K30, $20 \mathrm{mg}$ ) were dissolved in $43 \mathrm{~mL}$ of water. After the dissolved solution was stirred for $20 \mathrm{~min}$, a freshly prepared $\mathrm{NaBH}_{4}$ solution ( $20 \mathrm{mg}$ in $20 \mathrm{~mL}$ deionized water) was then added quickly with vigorous stirring. Then, $10 \mathrm{~mL} \mathrm{~K}_{2} \mathrm{PtCl}_{6}(6 \mathrm{mM})$ was added into the mixture solution, followed by a stirring process for $20 \mathrm{~min}$. Pt-based hollow nanostructures were collected by further centrifugal separation $(16,000 \mathrm{rpm})$ and washed several times with water and ethanol. The final black powder CoPt sample was then obtained by oven drying at $60^{\circ} \mathrm{C}$ for $20 \mathrm{~h}$.

The morphology of the products was characterised by Hitachi $\$ 4800$ scanning electron microscope (SEM, Hitachi Ltd., Tokyo, Japan) and JEOL 2200F transmission electron microscope (TEM, JEOL Ltd., Tokyo, Japan). The crystal structure and chemical composition of the products were characterised by energy-dispersive X-ray spectroscopy (EDS, EDAX Inc., Mahwah, NJ, USA) and electron energy loss spectroscopy (ELLs) with the TEM. For X-ray diffraction (XRD, PANalytical B.V., Almelo, Netherlands) measurements, powder samples were analysed with an X'Pert Pro MPD system ( $\mathrm{Cu} K \alpha$ X-ray source). The emission spectrum was observed with a Raman spectrometer (Horiba, Ltd., Kyoto, Japan), using a $504 \mathrm{~nm}$ wavelength excitation laser.

Pt-based hollow nanostructures in ethanol solution were dropped onto a silicon wafer and dried. Afterward, the substrate was immersed in $10^{-4} \mathrm{~mol} / \mathrm{L}$ PATP ethanol solution for $6 \mathrm{~h}$. Then the Si substrate, covered with the sample, was taken out and constantly rinsed by ethanol. After being dried in air, the substrate was characterised by SERS measurement. A Horiba Jobin Yvon Raman spectrometer (Horiba, Ltd., Kyoto, Japan) with $532 \mathrm{~nm}$ laser (laser spot size $1.5 \mu \mathrm{m}$ and beam power $320 \mu \mathrm{W})$ was used to measure the Raman spectra. SERS spectra were collected with a $50 \times$ objective lens. A total of 10 or 100 spectra were measured for an integration time of $20 \mathrm{~s}$ each, giving a total SERS measurement time of $200 \mathrm{~s}$ or $2000 \mathrm{~s}$, respectively. 


\section{Results}

The morphology of the as-prepared products was investigated by SEM at different magnifications. A typical low-magnification SEM image, as seen in in Figure 1a, shows that the sample can be prepared in uniform large quantities. The Pt-based hollow nanostructure size is about $300 \mathrm{~nm}$, as shown in Figure 1c,d. It demonstrates that the Pt-based hollow nanostructure surface is rough, indicating that the sample has a high surface area to volume ratio. This feature is very important for enhancing the substrate SERS activity, especially for the Pt substrate, which has low SERS activity, to allow the acquisition of high quality SERS spectra. The SERS and catalysing property of as-prepared Pt hollow nanostructures will be described later. The EDS data (Figure S1a) shows that the main signal peaks are ascribed to the elements $\mathrm{Co}$ and $\mathrm{Pt}$, and reveals that the atomic ratio of Co to $\mathrm{Pt}$ is nearly 30:70. The XRD data of samples is shown in Figure S1b, which represents a good match with the face-centered cubic (fcc) structure of CoPt, according to the Bragg reflections data [35,36].
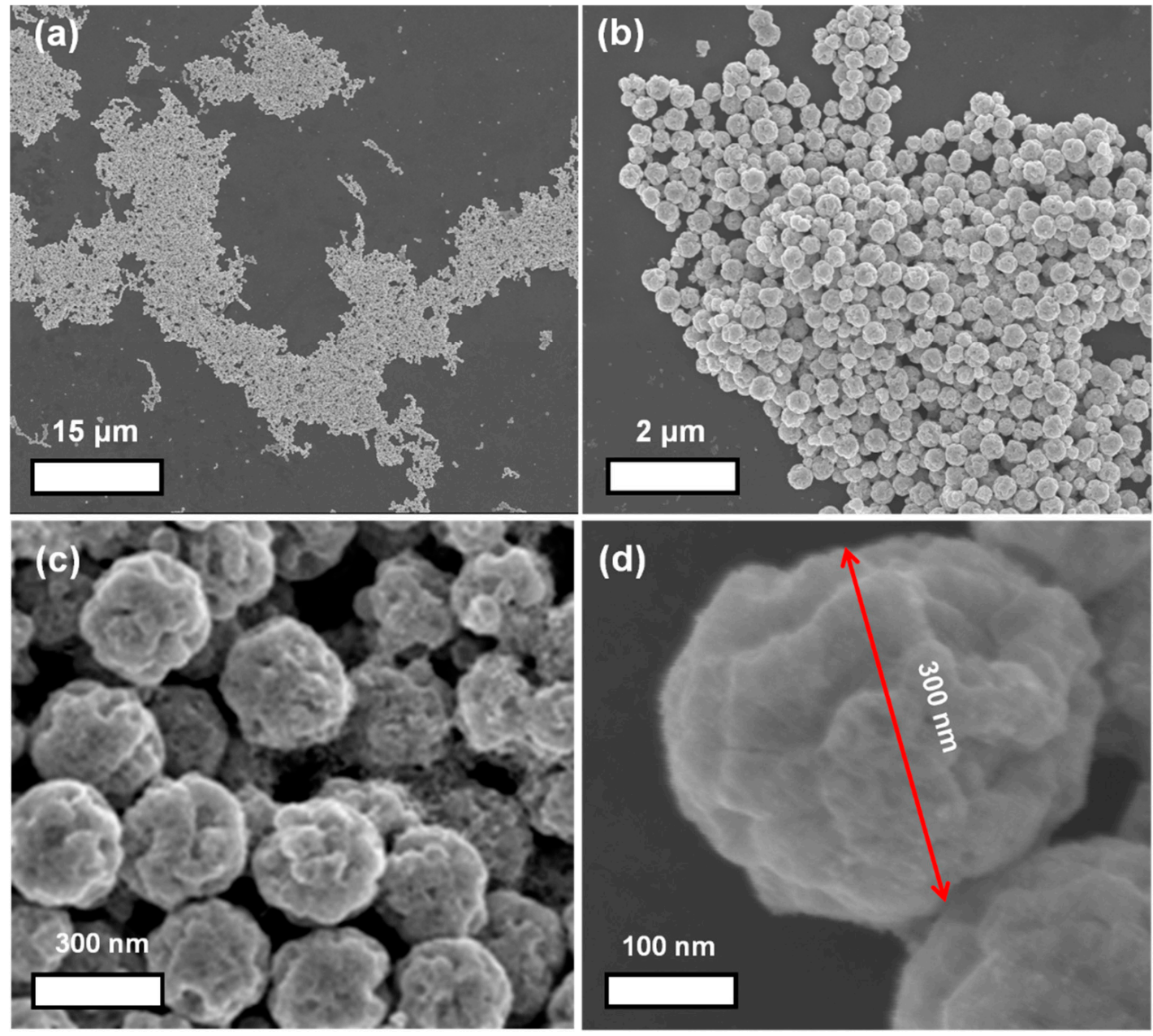

Figure 1. Scanning electron microscope (SEM) images of Pt-based hollow nanostructures. Scale bars in $(\mathbf{a}-\mathbf{d})$ indicate the images proceed from from low to high magnification.

The TEM image shown in Figure 2a displays a single Pt-based nanostructure, in which a hollow core can be deduced from the distinct color contrast. The size of the Pt-based hollow nanostructure is about $300 \mathrm{~nm}$, in agreement with the SEM image in Figure 1d. In Figure 2b, the hollow structure was more evident given the bright colour contrast. To investigate the elemental distribution of the Pt-based hollow nanostructures, the TEM-EELs Co mapped image of the Pt-based hollow nanostructure is shown in Figure 2c. Because the EELs spectroscopy is not sensitive to the Pt element, and no EELs Pt 
map could be collected. The EELs spectrum of Co is shown in Figure S2. The corresponding selected area electron diffraction (SAED) pattern taken from the area shown in Figure 2a, demonstrates a typical polycrystalline fcc structure with lattice parameter $a=0.38 \mathrm{~nm}$. It is in good agreement with the obtained XRD data, and the lattice fringes of CoPt which were measured to be 0.22 and $0.19 \mathrm{~nm}$, corresponding to the (111) and (200) planes, respectively. High-resolution TEM (HR-TEM) images of the Pt-based hollow nanostructures are shown in Figure S3 for additional support.
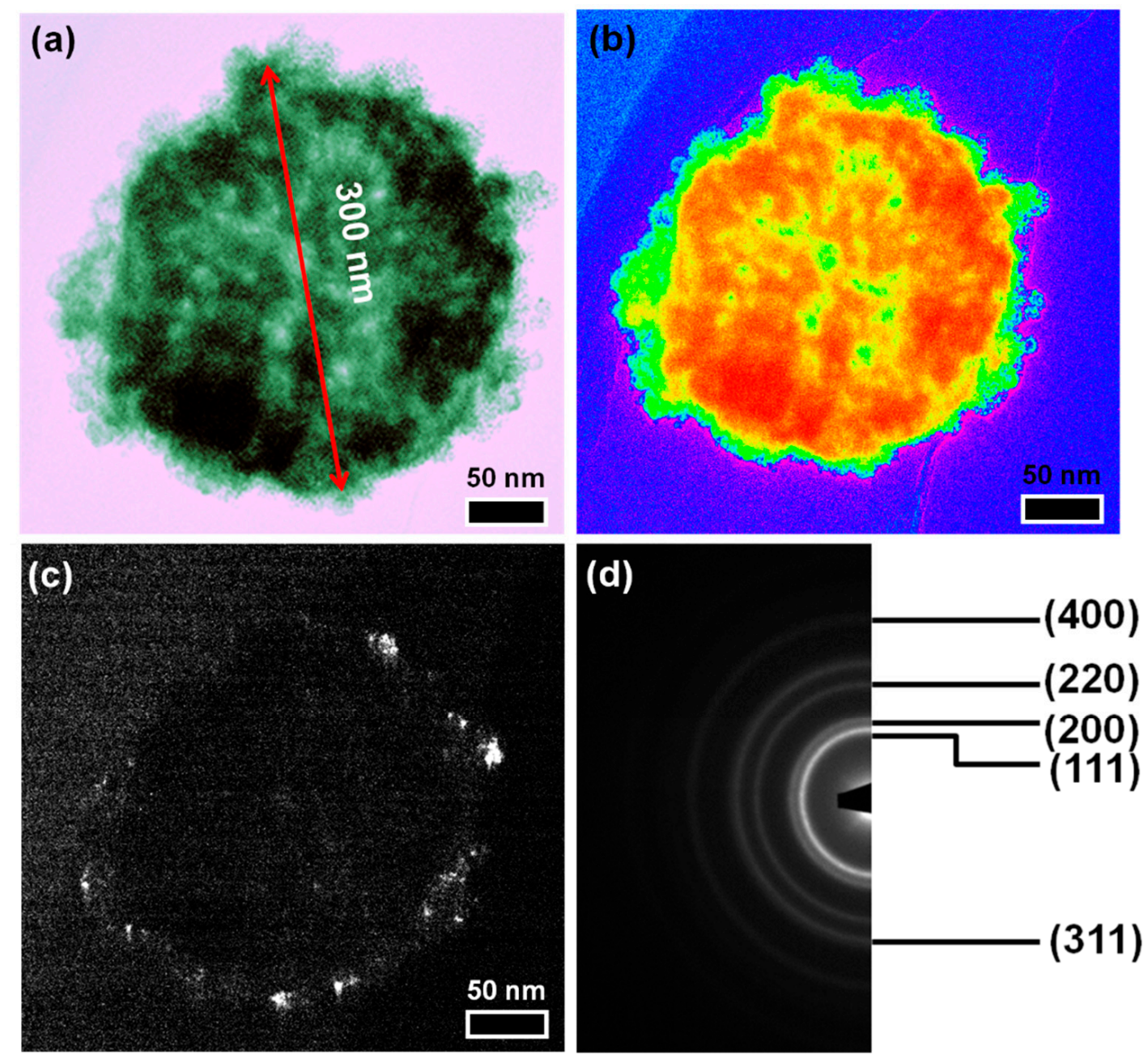

Figure 2. (a) Transmission electron microscope (TEM) image of a single Pt-based hollow nanostructure. (b) TEM image of a single Pt-based hollow nanostructure with enhance color contrast. (c) TEM-EELs mapping of Co element in the Pt-based hollow nanostructure. (d) The corresponding selected area electron diffraction (SAED) image of Pt-based hollow nanostructure.

The genuine SERS spectrum of the $10^{-4} \mathrm{~mol} / \mathrm{L}$ PATP molecules [37] adsorbed on the Pt-based hollow nanostructure surfaces under a laser wavelength of $532 \mathrm{~nm}$ is shown in Figure 3a. The emission spectrum of the Pt nanostructure powder was observed with a Raman spectrometer, using a $504 \mathrm{~nm}$ wavelength excitation laser as shown in Figure S4. In the SERS spectrum, the band at $1580 \mathrm{~cm}^{-1}$ is assigned to the $C=C$ stretching vibration of the benzenyl ring mode and the band at $1080 \mathrm{~cm}^{-1}$ is attributed to the C-S stretching mode. The band at $1180 \mathrm{~cm}^{-1}$ corresponds to the C-H deformation mode [31]. And it can be calculated that the EF factor is approximately $1 \times 10^{6}$, and the detailed analysis method can be seen in the Supporting Information (Figure S5). In addition, the high power of the laser on the focal spot can result in the thermal effects. This is known to promote the PATP dimerising to DMAB. However, as shown in Figure 3a, the vibrational peaks of DMAB at 1140, 1390, and $1432 \mathrm{~cm}^{-1}$ 
were not observed in the SERS spectrum. This demonstrated that the SERS signal could be attributed to the PATP monomer, instead of DMAB dimerising from two PATP molecules. In addition, owing to the thermal effects caused under prolonged illumination time, the time dependence of SERS spectra needed to be investigated to demonstrate the stability of the substrate for acquiring a genuine PATP SERS spectrum. Figure $3 \mathrm{~b}$ gives the time dependence of SERS spectra for PATP molecules at the very beginning and after $200 \mathrm{~s}$ of continuous laser irradiation on the substrate. The bands from DMAB molecules at 1140,1390 , and $1432 \mathrm{~cm}^{-1}$ ascribed to b2 mode were still not observed. This was able to exclude the plasmon-driven chemical conversion of PATP to DMAB under extended illumination time, as no spectral changes were observed in the experiment. The mechanism could be related to the highly-damping optical response of the Pt material, since the Pt-based hollow nanostructures exhibited weak dimerising effects with PATP molecules.

(a)

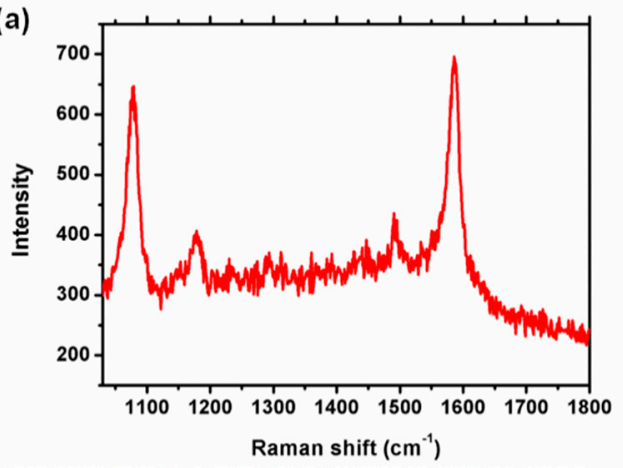

(b)

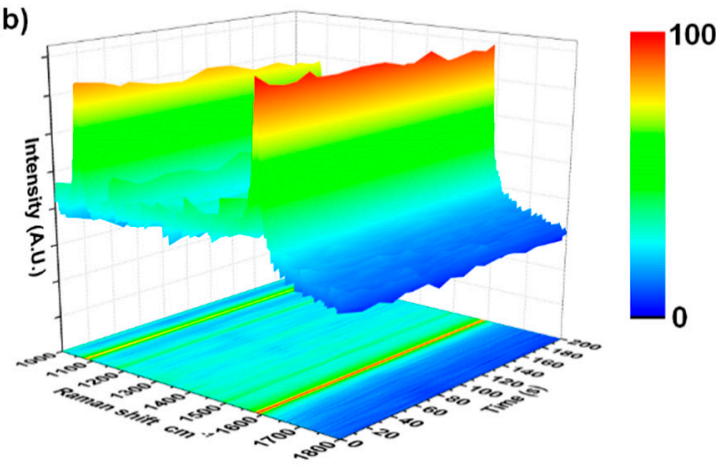

Figure 3. (a) Surface enhanced Raman scattering (SERS) spectrum of $10^{-4} \mathrm{~mol} / \mathrm{L}$ PATP molecules measured with $40 \mathrm{~s}$ of laser irradiating on the Pt-based hollow nanostructure substrate. (b) Time-dependent SERS spectra of $10^{-4} \mathrm{~mol} / \mathrm{L}$ PATP on the Pt-based hollow nanostructure substrate illuminated with the laser power of $0.3 \mathrm{~mW}$.

\section{Discussion}

Problems may result from the illumination time dependence in combination with variations to tip molecule concentration, therefore this was also taken into account in the SERS experiments. To further illustrate the stability of the substrate for ascertaining genuine PATP SERS spectrum, the concentration of PATP molecules was varied, while maintaining the increased the illumination time. Figure $4 \mathrm{a}-\mathrm{c}$ display time dependence of SERS spectra (200 s) with different tip molecule concentrations at $10^{-5}$, $10^{-6}$, and $10^{-7} \mathrm{~mol} / \mathrm{L}$. It shows that the spectra were stable and no new peaks (at 1140, 1390, and $1432 \mathrm{~cm}^{-1}$ ) attributed to DMAB emerged with PATP concentration changes. Figure $4 \mathrm{~d}-\mathrm{f}$ reveal the time dependence of SERS spectra with PATP molecule concentrations at $10^{-5}, 10^{-6}$ and $10^{-7} \mathrm{~mol} / \mathrm{L}$, for which the illumination time was increased from $200 \mathrm{~s}$ to $2000 \mathrm{~s}$. By comparing with the results from Figure $4 \mathrm{a}-\mathrm{c}$, it can be seen that plasmon-driven chemical conversion of PATP to DMAB was not occurring, even though the peak intensity of SERS spectra continuously decreased. Thus, the results demonstrate that the plasmon-driven chemical conversion was suppressed and the substrate is stable under laser illumination. 

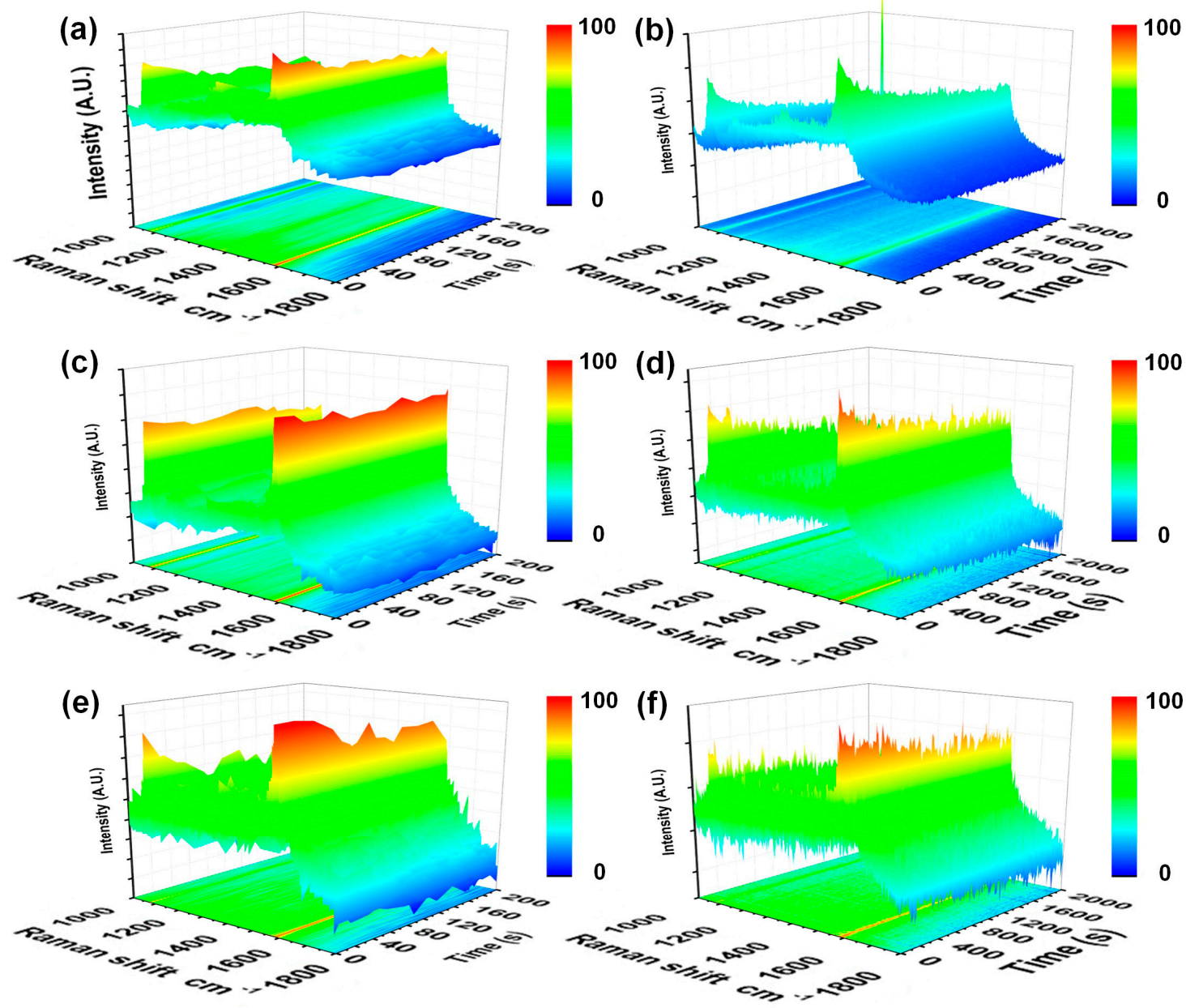

Figure 4. Time-dependent SERS spectra of Pt-based hollow nanostructures on Si, after adsorption of PATP from an ethanol solution, with different laser illumination time. The concentration of PATP in solution and laser irradiation time for collecting Raman spectra from these samples are: (a) $10^{-5} \mathrm{M}$, $200 \mathrm{~s}$; (b) $10^{-5}$ M, $2000 \mathrm{~s}$; (c) $10^{-6}$ M, $200 \mathrm{~s}$; (d) $10^{-6}$ M, $2000 \mathrm{~s}$; (e) $10^{-7}$ M, $200 \mathrm{~s}$; (f) $10^{-7}$ M, $2000 \mathrm{~s}$.

\section{Conclusions}

In summary, genuine SERS spectra of PATP molecules were successfully observed with Pt-based hollow nanostructures, though Pt itself has weak SERS activity. In the reported SERS measurements, PATP molecule dimerisation did not occur, and vibrational modes of 1140, 1390 and $1432 \mathrm{~cm}^{-1}$ related to DMAB molecules were not observed. The SERS spectra are in good agreement with the Raman spectrum of PATP powder, even the laser illumination time is prolonged. The laser illumination time and the concentration of PATP molecules in solution are both the key factors that affect the plasmon-driven chemical conversion from PATP to DMAB, due to the thermal effects of a laser focal spot. In this work, the illumination time and the tip molecule concentration show no influence on the isolated SERS spectra of PATP, demonstrating the stable SERS catalytic stability of Pt-based hollow nanostructures. Furthermore, this suggests that the substrate with its unique optical properties may be useful in other SERS detection processes to obtain genuine SERS spectra.

Supplementary Materials: Supplementary materials are available online at http:/ www.mdpi.com/2076-3417/ 7/9/953/s1.

Acknowledgments: This work was supported by the National Natural Science Foundation of China (NO. 51371015, 51331002, 11674023 and 21601013) and Beijing Municipal Science and Technology Project (NO. Z17111000220000). 
Author Contributions: Yinong Wang, Yinghui Sun and Rongming Wang conceived and designed the experiments; Yinong Wang, Weihua Lin, Huanyu Ye, En Cao and Yinghui Sun performed the experiments and analyzed the data; Haofei Zhao, En Cao Yinghui Sun and Rongming Wang wrote the paper; Haofei Zhao, Cao En, Weihua Lin and Huanyu Ye participated in paper revision and made many suggestions. All authors read and approved the final manuscript. Yinong Wang and Haofei Zhao contributed equally to this work.

Conflicts of Interest: The authors declare no conflict of interest.

\section{References}

1. Puntes, V.F. Colloidal Nanocrystal Shape and Size Control: The Case of Cobalt. Science 2001, 291, $2115-2117$. [CrossRef] [PubMed]

2. Ma, Y.; Oleynikov, P.; Terasaki, O. Electron crystallography for determining the handedness of a chiral zeolite nanocrystal. Nat. Mater. 2017, 16, 755-759. [CrossRef] [PubMed]

3. Sun, S. Monodisperse FePt Nanoparticles and Ferromagnetic FePt Nanocrystal Superlattices. Science 2000, 287, 1989-1992. [CrossRef] [PubMed]

4. Klimov, V.I. Optical Gain and Stimulated Emission in Nanocrystal Quantum Dots. Science 2000, 290, 314-317. [CrossRef] [PubMed]

5. Pietryga, J.M.; Park, Y.S.; Lim, J.; Fidler, A.F.; Bae, W.K.; Brovelli, S.; Klimov, V.I. Spectroscopic and Device Aspects of Nanocrystal Quantum Dots. Chem. Rev. 2016, 116, 10513-10622. [CrossRef] [PubMed]

6. Shan, A.; Chen, Z.; Li, B.; Chen, C.; Wang, R. Monodispersed, ultrathin NiPt hollow nanospheres with tunable diameter and composition via a green chemical synthesis. J. Mater. Chem. A 2015, 3, 1031-1036. [CrossRef]

7. Gan, L.; Rudi, S.; Cui, C.; Heggen, M.; Strasser, P. Size-Controlled Synthesis of Sub-10 nm PtNi3 Alloy Nanoparticles and their Unusual Volcano-Shaped Size Effect on ORR Electrocatalysis. Small 2016, 12, 3189-3196. [CrossRef] [PubMed]

8. Wang, Y.; Wang, H.; Shen, Y.; Xu, S.; Xu, W. Plasmon-Driven Dynamic Response of a Hierarchically Structural Silver-Decorated Nanorod Array for Sub-10 nm Nanogaps. ACS Appl. Mater. Interfaces 2016, 8, 15623-15629. [CrossRef] [PubMed]

9. Li, J.; Chen, C.; Jans, H.; Xu, X.; Verellen, N.; Vos, I.; Okumura, Y.; Moshchalkov, V.V.; Lagae, L.; Van Dorpe, P. $300 \mathrm{~mm}$ Wafer-level, ultra-dense arrays of Au-capped nanopillars with sub-10 nm gaps as reliable SERS substrates. Nanoscale 2014, 6, 12391-12396. [CrossRef] [PubMed]

10. Ding, Q.; Shi, Y.; Chen, M.; Li, H.; Yang, X.; Qu, Y.; Liang, W.; Sun, M. Ultrafast Dynamics of Plasmon-Exciton Interaction of Ag Nanowire- Graphene Hybrids for Surface Catalytic Reactions. Sci. Rep. 2016, 6, 32724. [CrossRef] [PubMed]

11. Yang, X.; Yu, H.; Guo, X.; Ding, Q.; Pullerits, T.; Wang, R.; Zhang, G.; Liang, W.; Sun, M. Plasmon-exciton coupling of monolayer MoS 2-Ag nanoparticles hybrids for surface catalytic reaction. Mater. Today Energy 2017, 5, 72-78. [CrossRef]

12. Tang, S.; Chen, M.; Zheng, N. Sub-10-nm Pd nanosheets with renal clearance for efficient near-infrared photothermal cancer therapy. Small 2014, 10, 3139-3144. [CrossRef] [PubMed]

13. Zhang, N.; Han, C.; Xu, Y.-J.; Foley, J.J., IV; Zhang, D.; Codrington, J.; Gray, S.K.; Sun, Y. Near-field dielectric scattering promotes optical absorption by platinum nanoparticles. Nat. Photonics 2016, 10, 473-482. [CrossRef]

14. Chen, J.; Wiley, B.; McLellan, J.; Xiong, Y.; Li, Z.Y.; Xia, Y. Optical properties of Pd-Ag and Pt-Ag nanoboxes synthesized via galvanic replacement reactions. Nano Lett. 2005, 5, 2058-2062. [CrossRef] [PubMed]

15. Li, J.F.; Zhang, Y.J.; Ding, S.Y.; Panneerselvam, R.; Tian, Z.Q. Core-Shell Nanoparticle-Enhanced Raman Spectroscopy. Chem. Rev. 2017, 117, 5002-5069. [CrossRef] [PubMed]

16. Ban, R.; Yu, Y.; Zhang, M.; Yin, J.; Xu, B.; Wu, D.Y.; Wu, M.; Zhang, Z.; Tai, H.; Li, J.; et al. Synergetic SERS Enhancement in a Metal-Like/Metal Double-Shell Structure for Sensitive and Stable Application. ACS Appl. Mater. Interfaces 2017, 9, 13564-13570. [CrossRef] [PubMed]

17. Shin, J.H.; Kim, H.G.; Baek, G.M.; Kim, R.; Jeon, S.; Mun, J.H.; Lee, H.-B.-R.; Jung, Y.S.; Kim, S.O.; Kim, K.N.; et al. Fabrication of $50 \mathrm{~nm}$ scale Pt nanostructures by block copolymer (BCP) and its characteristics of surface-enhanced Raman scattering (SERS). RSC Adv. 2016, 6, 70756-70762. [CrossRef] 
18. Liu, L.; Yang, H.; Ren, X.; Tang, J.; Li, Y.; Zhang, X.; Cheng, Z. Au-ZnO hybrid nanoparticles exhibiting strong charge-transfer-induced SERS for recyclable SERS-active substrates. Nanoscale 2015, 7, 5147-5151. [CrossRef] [PubMed]

19. Li, J.F.; Huang, Y.F.; Ding, Y.; Yang, Z.L.; Li, S.B.; Zhou, X.S.; Fan, F.R.; Zhang, W.; Zhou, Z.Y.; WuDe, Y.; et al. Shell-isolated nanoparticle-enhanced Raman spectroscopy. Nature 2010, 464, 392-395. [CrossRef] [PubMed]

20. Fleischmann, M.; Hendra, P.J.; McQuillan, A.J. Raman spectra of pyridine adsorbed at a silver electrode. Chem. Phys. Lett. 1974, 26, 163-166. [CrossRef]

21. Anema, J.R.; Li, J.F.; Yang, Z.L.; Ren, B.; Tian, Z.Q. Shell-Isolated Nanoparticle-Enhanced Raman Spectroscopy: Expanding the Versatility of Surface-Enhanced Raman Scattering. Annu. Rev. Anal. Chem. 2011, 4, 129-150. [CrossRef] [PubMed]

22. Duan, S.; Wang, R. Bimetallic nanostructures with magnetic and noble metals and their physicochemical applications. Prog. Nat. Sci. Mater. Int. 2013, 23, 113-126. [CrossRef]

23. Xia, T.; Liu, J.; Wang, S.; Wang, C.; Sun, Y.; Wang, R. Nanomagnetic CoPt truncated octahedrons: Facile synthesis, superior electrocatalytic activity and stability for methanol oxidation. Sci. China Mater. 2016, 60, 57-67. [CrossRef]

24. Liu, J.; Xia, T.; Wang, S.; Yang, G.; Dong, B.; Wang, C.; Ma, Q.; Sun, Y.; Wang, R. Oriented-assembly of hollow FePt nanochains with tunable catalytic and magnetic properties. Nanoscale 2016, 8, 11432-11440. [CrossRef] [PubMed]

25. Choi, S.; Kweon, S.; Kim, J. Electrodeposition of Pt nanostructures with reproducible SERS activity and superhydrophobicity. Phys. Chem. Chem. Phys. 2015, 17, 23547-23553. [CrossRef] [PubMed]

26. Choi, S.; Jeong, H.; Choi, K.H.; Song, J.Y.; Kim, J. Electrodeposition of triangular Pd rod nanostructures and their electrocatalytic and SERS activities. ACS Appl. Mater. Interfaces 2014, 6, 3002-3007. [CrossRef] [PubMed]

27. Liu, R.; Liu, J.F.; Zhang, Z.M.; Zhang, L.Q.; Sun, J.F.; Sun, M.T.; Jiang, G.B. Submonolayer-Pt-Coated Ultrathin Au Nanowires and Their Self-Organized Nanoporous Film: SERS and Catalysis Active Substrates for Operando SERS Monitoring of Catalytic Reactions. J. Phys. Chem. Lett. 2014, 5, 969-975. [CrossRef] [PubMed]

28. Muniz-Miranda, M.; Pergolese, B.; Muniz-Miranda, F.; Caporali, S. SERS effect from Pd surfaces coated with thin films of Ag colloidal nanoparticles. J. Alloy. Compd. 2014, 615, S357-S360. [CrossRef]

29. Sun, Q.; Wang, S.; Wang, R. Well-Aligned CoPt Hollow Nanochains Synthesized in Water at Room Temperature. J. Phys. Chem. C 2012, 116, 5352-5357. [CrossRef]

30. Sun, Q.; Ren, Z.; Wang, R.; Wang, N.; Cao, X. Platinum catalyzed growth of NiPt hollow spheres with an ultrathin shell. J. Mater. Chem. 2011, 21, 1925-1930. [CrossRef]

31. Tian, X.; Chen, L.; Xu, H.; Sun, M. Ascertaining genuine SERS spectra of $p$-aminothiophenol. RSC Adv. 2012, 2, 8289-8292. [CrossRef]

32. Gao, Y.; Yang, N.; You, T.; Jiang, L.; Yin, P. Ultra-thin Au tip structure: A novel SERS substrate for in situ observation of a $p$-aminothiophenol surface-catalytic reaction. RSC Adv. 2017, 7, 4541-4546. [CrossRef]

33. Fang, Y.; Li, Y.; Xu, H.; Sun, M. Ascertaining $p, p^{\prime}$-dimercaptoazobenzene produced from $p$-aminothiophenol by selective catalytic coupling reaction on silver nanoparticles. Langmuir 2010, 26, 7737-7746. [CrossRef] [PubMed]

34. Ikeda, K.; Sato, J.; Fujimoto, N.; Hayazawa, N.; Kawata, S.; Uosaki, K. Plasmonic Enhancement of Raman Scattering on Non-SERS-Active Platinum Substratest. J. Phys. Chem. C 2009, 113, 11816-11821. [CrossRef]

35. Tzitzios, V.; Niarchos, D.; Margariti, G.; Fidler, J.; Petridis, D. Synthesis of CoPt nanoparticles by a modified polyol method: Characterization and magnetic properties. Nanotechnology 2005, 16, 287. [CrossRef] [PubMed]

36. Mallet, J.; Yu-Zhang, K.; Chien, C.-L.; Eagleton, T.S.; Searson, P.C. Fabrication and magnetic properties of fcc CoXPt1-X nanowires. Appl. Phys. Lett. 2004, 84, 3900-3902. [CrossRef]

37. Santoro, G.; Yu, S.; Schwartzkopf, M.; Zhang, P.; Koyiloth Vayalil, S.; Risch, J.F.H.; Rübhausen, M.A.; Hernández, M.; Domingo, C.; Roth, S.V. Silver substrates for surface enhanced Raman scattering: Correlation between nanostructure and Raman scattering enhancement. Appl. Phys. Lett. 2014, 104, 243107. [CrossRef]

(C) 2017 by the authors. Licensee MDPI, Basel, Switzerland. This article is an open access article distributed under the terms and conditions of the Creative Commons Attribution (CC BY) license (http://creativecommons.org/licenses/by/4.0/). 